P188 (continued)

Results: Initial findings suggest common achievements included developing school gardens and establishing multi-component education programs (such as 'Harvest of the Month') while challenges related to staff turnover and lack of dedicated personnel resources. School districts often described lessons learned in terms of increasing program outreach and sustainability by developing core activity areas.

Conclusions and Implications: Findings from this study inform support services for future grant recipients and enable OCFS to further develop resources for partnering school districts, state and local agencies, tribal organizations, and non-profit entities. Results will also be used to describe effective practices for other schools planning and implementing farm to school activities.

Funding: USDA

\section{P189 Technology Use and Preferences of EFNEP Paraprofessional Educators}

Mira Mehta,PhD, mmehta@umd.edu, University of Maryland, College Park, 0102 Skinner Building, 4300 Chapel Lane, University of Maryland, College Park, MD 20742; Beth Peralta, MS, RD, University of Illinois, UrbanaChampaign; Kavitha Sankavaram, PhD, University of Maryland, College Park; Lisa Martin, MS, RD, Kansas State University; Joan D. Paddock, MPH, BS, Cornell University; Jennifer McCaffrey, PhD, MPH, RD, University of Illinois, Urbana-Champaign

Objective: To understand the usage of technology by paraprofessional educators providing direct nutrition education to low income families/youth in the Expanded Food and Nutrition Education Program (EFNEP).

Study Design, Settings, Participants: EFNEP paraprofessional educators serving low income communities in Maryland, Illinois and Kansas.

Outcome Measures and Analysis: Data was gathered regarding technology use, frequency of use, location where accessed, preferred platforms, reason for accessing different devices as well as demographic data.

Results: Sixty-two EFNEP educators from three states completed the surveys. Technology use and preferences differed considerably between states and sample size was not representative of the nation. Eighty-eight percent of educators accessed a computer daily from their place of work; $92 \%$ used their mobile device for texting and 73\% used a Smartphone to download and use applications. A majority (>90\%) had access to high-speed internet to access nutrition information about healthy eating, recipes and exercise. Eighty-five of educators also used social media with Facebook, Pinterest and YouTube representing the most popular sites.

Conclusions and Implications: EFNEP has a proven record of cost-effective direct nutrition education in a series to food insecure communities across all states/territories in the United States using an 'indigenous paraprofessional' model for program delivery. Working families with children face numerous challenges in engaging in repeated face to face education. This study aims to understand technology preferences and use by EFNEP educators in order to improve retention and graduation from EFNEP. This study can inform efforts to examine the feasibility of strategic and limited use of technology to augment EFNEP nutrition education while maintaining the integrity of EFNEP's direct education model.

Funding: None

\section{P190 Telephonic Health Coaching (THC) Promotes Health Behavior Changes Among Participants in SNAP-Ed}

Meg Gaughan, PhD, RD, mgaughan@usj.edu, University of Saint Joseph, 1678 Asylum Avenue, West Hartford, CT 06117; Debbie Brinckman, MS, RDN, CD-N, University of Saint Joseph

Objective: To develop and test the efficacy of a THC program for the SNAP-Ed population.

Theory, Prior Research, Rationale: THC is a feasible and cost-effective method used to promote a spectrum of health behavior changes. In 2015, we launched a THC program with SNAP-Ed clients to pilot test procedures, methods, and evaluate preliminary outcomes. The current THC protocol employs a Brief Action Planning model; a theory-based approach that incorporates motivational interviewing techniques, building self-efficacy, and setting SMART goals.

Description: The target population is SNAP-Ed attendees from various community sites. Clients signed informed consent and provided contact information. Clients were called for a 15 minute THC session, weekly for 6 weeks. At week 1 and 6 clients completed a 12-item survey on eating and physical activity behaviors based on the Dietary Guidelines (DG). Clients set 1-2 goal per session. Behavior change was measured by self-reported percent attainment (0\%-100\%) of goals, and survey results.

Evaluation: Fifty-seven clients began THC; 34 (60\%) completed week 4, and 27 (47\%) completed all 6 weeks. Goals relating to increasing physical activity (37\%) and fruit and vegetable intake (25\%) dominated. Clients reported attaining $100 \%$ of their goals $49 \%$ of the time. Ninety-three percent of the clients made a significant positive behavior change in one of the DG. Direct cost of THC was sustainable at $\$ 10 /$ session.

Conclusions and Implications: $\mathrm{THC}$ is an innovative mode of promoting healthy behaviors with a subset of highly motivated SNAP-Ed clients. It has the potential to extend resources, impact a broader geographic range, and offer a more client-focused interaction of greater duration to facilitate behavior change.

Funding: Supplemental Nutrition Assistance Program Education 